\title{
FINE STRUCTURE OF THE SPERM HEAD IN EJACULATE AND UTERINE SPERMATOZOA OF THE RÄBBIT
}

\author{
J. M. BEDFORD \\ Department of Physiology, Royal Veterinary College, London, N.W.1
}

(Received 4th September 1963)

\begin{abstract}
Summary. The microstructure of the rabbit sperm head in ejaculate and in uterine spermatozoa has been examined with the electron microscope. The normal structure of the sperm head is described, and it is suggested that the properties of the plasma membrane may vary over different regions. No morphological change in the acrosome cap, which might account for the functional difference in capacitated spermatozoa, has been observed. There are indications, however, that the plasma membrane is more easily separable from the anterior and lateral edges of the underlying acrosome cap in spermatozoa recovered from the uterus.

Abnormal forms of acrosome cap development are described, and are discussed particularly in relation to similar abnormalities described previously in bull and boar spermatozoa.
\end{abstract}

\section{INTRODUCTION}

A functional change, termed capacitation, occurs in rabbit and in rat spermatozoa during passage through the female tract (Austin, 1951; Chang, 1951). As yet no difference has been found in uterine spermatozoa which can unequivocally be attributed to the capacitation process, although it has been suggested that capacitation may possibly be associated with an increase in sperm respiration rate (Hamner \& Williams, 1963). Austin \& Bishop (1958b) observed that in guinea-pigs and in certain rodents the acrosome cap was lost from those spermatozoa seen to be penetrating the zona pellucida, lying in the perivitelline space, or entering the vitellus: this observation has been confirmed by Ohnuki (1959) in the mouse and by Moricard (1960), Hadek (1963) and Bedford (1963) in the rabbit. Visible changes in the acrosome have been observed in some of the spermatozoa recovered from the uterine and tubal lumen in these species, and this observation has prompted the suggestion that acrosomal changes seen in spermatozoa recovered from the female tract may constitute a morphological concomitant of the capacitation process (Austin \& Bishop, 1958b; Ohnuki, 1959; Austin, 1961). Notwithstanding these suggestions, the results of several experiments with rabbit spermatozoa do not appear to support such a hypothesis for this species, in which it seems more likely that the 
acrosome changes seen prior to fertilization are manifestations of some degenerative process (Bedford, 1963). Nevertheless, in view of the manifest functional difference between ejaculate and capacitated spermatozoa, it seemed reasonable that the process of capacitation might involve structural changes not discernible with the light microscope. For this reason the morphological features of the sperm head in samples of rabbit ejaculate and uterine spermatozoa have been examined with the electron microscope. Certain aspects of sperm head structure have also been included in this report, since no detailed accounts of the fine structure of rabbit spermatozoa appear to have been published up to the present time.

\section{MATERIALS AND METHODS}

Ejaculate spermatozoa were obtained with an artificial vagina, from fertile males of mixed breed. Some were fixed immediately after collection, while some were incubated in Ringer's solution containing $10 \%$ homologous heated serum at $37^{\circ} \mathrm{C}$ for $12 \mathrm{hr}$ before fixation. Uterine spermatozoa were obtained by flushing the uterine horns of mated females with about $2 \mathrm{ml}$ of Ringer's solution, some $10 \mathrm{hr}$ after mating. Both ejaculate and uterine spermatozoa were then treated as follows: sperm samples were centrifuged at $1500 \mathrm{rev} / \mathrm{min}$ for 15 min, after which the supernatant fluids were decanted. The precipitate pellets containing the spermatozoa were then fixed for about $2 \mathrm{hr}$ at $10^{\circ} \mathrm{G}$ in $1 \%$ osmium tetroxide in veronal acetate buffer, $\mathrm{pH} 7.4$ (Palade, 1952), with the addition of $0.25 \mathrm{~m}$-sucrose (Caulfield, 1957). After fixation the pellets were immersed in $30 \%$ methanol and dehydrated in ascending concentrations of methanol up to $100 \%$ during a period of $1 \mathrm{hr}$, followed by immersion in acetone for $2 \mathrm{hr}$. After embedding in Araldite (Glauert \& Glauert, 1958), polymerized at $60^{\circ} \mathrm{C}$ in gelatin capsules, sections of approximately $0.08 \mu$ thickness were cut with glass knives on a Cambridge (Huxley) microtome. These sections were stained either with lead hydroxide (Millonig, 1961) for about $30 \mathrm{~min}$, or with uranyl acetate followed by potassium permanganate, according to a technique developed by Dr A. M. Lawn (personal communication). The sections were examined with an R.C.A. EMU $3 F$ electron microscope.

\section{OBSERVATIONS}

EJACULATE SPERMATOZOA

The sperm nucleus tends to taper in the anterior region and has shown no evidence of structural organization (Pl. 1, Fig. 1). Small vacuoles, as reported by Fawcett (1958) in the human and in other sperm, and indentations of the nuclear surface, have been seen in a few spermatozoa. The nucleus is not symmetrical and in transverse section shows a rather greater radius of curvature on one surface than on the other (PI. 1, Fig. 2). The nucleus is enclosed by a double membrane; external to the outer nuclear membrane lie two discrete structures, namely the acrosome cap, which covers the anterior two-thirds of the nucleus, and the post-nuclear cap which unites with the posterior border of the acrosome cap. The acrosome cap consists of an amorphous substance bounded by a limiting membrane. In lead-stained sections this membrane 
appears as a single unit (Pl. 1, Fig. 3a), whereas sections stained with uranyl acetate and potassium permanganate show the acrosome membrane as having a bilaminar structure (Pl. 1, Fig. 3b), presumably similar to that described for the acrosome membrane of the bull spermatozoon (Kojima, 1962). The thickness of the double membrane seen in Pl. 1, Fig. 3b is however only about $75 \AA$, and the distance between the two lines does not vary in the regions where they remain sharply defined. It therefore seems reasonable to regard the acrosome membrane functionally as a single membrane, within the terms of the 'unit' membrane theory of Robertson (1960).

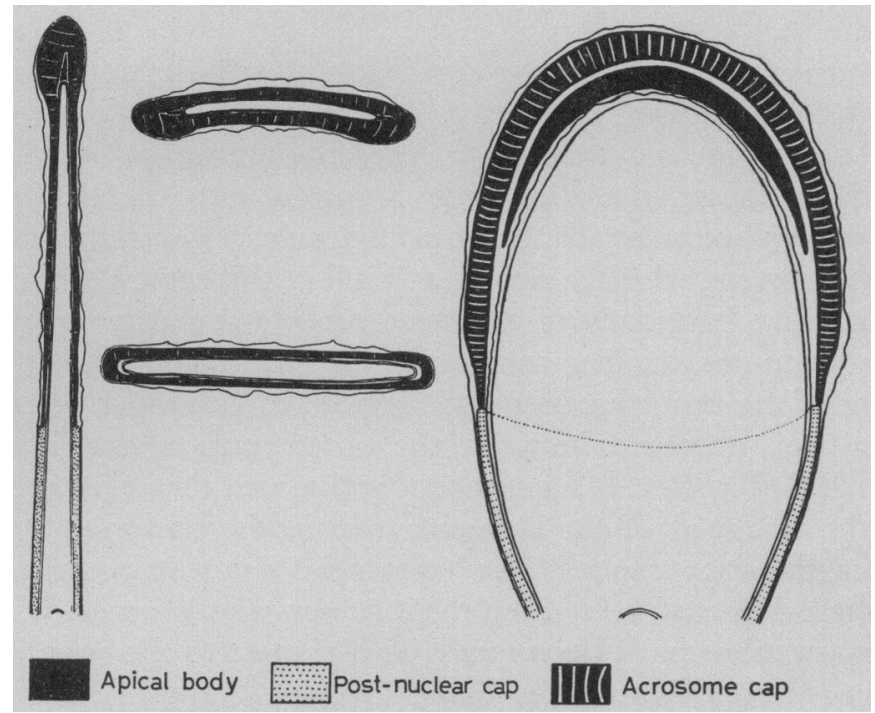

TeXT-FIG. 1. Diagrammatic representation of the structural components of the rabbit sperm head.

Sagittal (Pl. 1, Fig. 1) and transverse sections (Pl. 1, Fig. 2) of the sperm head show that the relative proportions of the acrosome cap vary in different regions. In the posterior region the cap appears as a relatively thin structure, whereas the anterior and antero-lateral regions are comparatively much thicker. In the anterior and antero-lateral regions the internal acrosome membrane assumes the form of a cone (Pl. 1, Fig. 4). The space within the elevated internal acrosome membrane appears to be filled with some electron dense material, the inner boundary of which is clearly delineated, and separate from the adjacent edge of the sperm nucleus. Observations on many sperm heads, cut at different angles, have shown that the material of this apical cone or body extends as a ridge along the anterior and antero-lateral edges of the sperm head, gradually tapering along the lateral edges to cease at some point anterior to the posterior border of the acrosome cap. The probable form of the whole structure is represented diagrammatically in Text-fig. I. In occasional spermatozoa the acrosome cap was seen to be disintegrating, but in each case the inner acrosome membrane and the apical body always remained intact (Pl. 2, Fig. 5). 
Sagittal sections of most spermatozoa have shown the anterior acrosome region as a smooth swelling. A few spermatozoa have been observed, in both ejaculate and uterine samples, which showed a variety of structural forms. In some an anterior flap was present (Pl. 2, Fig. 6) while others showed various forms of swelling in which the apical cone was bent away from the nuclear line. A few spermatozoa in several samples showed an organized cystic structure in the apical region, containing material of granular appearance (Pl. 2, Fig. 7). In the samples in which a few spermatozoa showed structural differences, the majority had a uniform structure and the males concerned seemed to exhibit normal fertility, as judged by fertilization trials in other experiments incidental to this study.

The structure of the post-nuclear cap is less clearly defined than that of the acrosome cap. The post-nuclear cap, stained either by lead or by uranyl acetate and potassium permanganate, presents a spongy appearance which contrasts with the denser material of the acrosome cap (Pl. 1, Figs. 1 and 3a). The outer limiting boundary of the post-nuclear cap lies immediately beneath the plasma membrane, which is closely applied in this region of the sperm head. The inner limiting boundary of the post-nuclear cap appears to have been provided by the outer nuclear membrane in this region (Pl. 1, Fig. 3a). In many sections of the posterior acrosome region, the presence of an amorphous material has been observed between the outer nuclear and inner acrosome membranes (Pl. 1, Fig. 3a). It seems possible that this material, and the marked decrease in the thickness of the acrosome cap in the posterior region, may be responsible for the appearance of the 'equatorial segment' seen in spermatozoa viewed by phase-contrast, or stained for instance with Giemsa or Rose Bengal. Similar interpretations have been made with respect to the equatorial segment in boar spermatozoa (Nicander \& Bane, 1962).

The sperm head is enclosed by a membrane which appears to be continuous with the outer membrane of the midpiece and tail. It is apparent that the relationship of this plasma membrane with the post-nuclear cap differs from its relationship with the surface of the acrosome cap. The plasma membrane appears always in close apposition to the external surface of the post-nuclear cap, whereas over the dorsal and ventral surfaces of the acrosome cap the membrane appears ballooned and free, or only loosely adherent, and is in close contact only over the lateral and anterior edges of the acrosome (Pl. 1, Figs. 1 and 2). Although the extreme balloon effect of the membrane over the acrosome region in free spermatozoa may be due partially to fixation procedures, the difference in reaction of this membrane in different regions of the sperm head is nevertheless quite clear.

Sections of spermatozoa fixed after incubation at $37^{\circ} \mathrm{C}$ for about $12 \mathrm{hr}$ showed no difference in the morphology of the structures of the head, as compared with spermatozoa fixed immediately after ejaculation.

UTERINE SPERMATOZOA

The uterine contents taken $10 \mathrm{hr}$ after mating, contained free spermatozoa and spermatozoa in various stages of phagocytosis by uterine polymorpho- 
nuclear leucocytes. (Certain aspects of the phagocytosis of spermatozoa will be discussed in a separate communication.)

No structural difference in uterine spermatozoa has been clearly revealed as a concomitant of the functional change which occurs during capacitation. The plasma membrane and acrosome cap remained intact in a majority of uterine spermatozoa. Occasional spermatozoa did show various degrees of acrosome degeneration, but in all of these the inner acrosome membrane and apical cone remained intact, as in the degenerating ejaculate spermatozoa described previously (P1. 2, Fig. 5). There are some indications, however, of a change in the plasma membrane/acrosome relationship in uterine spermatozoa. In all ejaculate spermatozoa examined, including those incubated for $12 \mathrm{hr}$ before fixing, which in many cases were clearly adherent to each other by the plasma membrane over the acrosome region, the plasma membrane remained in close apposition to the anterior and lateral edges of the acrosome cap. In many uterine spermatozoa, on the other hand, the plasma membrane was widely separated from the acrosome edges, particularly in those spermatozoa adhering to white cells (Pl. 2, Fig. 8).

The adhesion of spermatozoa to leucocytes by the plasma membrane, is inferred from many micrographs in which uterine spermatozoa in close proximity to leucocytes, showed distortion of the plasma membrane over the acrosome region, where this membrane had come to lie in close apposition to, and to follow the contour of, the white cell membrane (Pl. 2, Fig. 6). In uterine spermatozoa the outer surface of the plasma membrane over the acrosome region commonly showed the presence of small particles apposed to and probably adherent to the membrane.

\section{DISCUSSION}

These observations confirm previous conclusions drawn from observations with the light microscope (Adams \& Chang, 1962; Bedford, 1963), and do not support the idea that visible structural changes in the acrosome accompany the capacitation process in rabbit spermatozoa. Some evidence has been obtained that the nature of the attachment of the plasma membrane to the edges of the acrosome cap may be changed in many uterine spermatozoa, such that this membrane is more easily separable from the acrosome at the anterior and lateral border. As yet, however, in view of the present lack of information concerning the role and fate of the superficial structures of the mammalian spermatozoa during the initial phase of the sperm/egg interaction, the significance of a change of this nature cannot easily be interpreted.

The structure of the rabbit sperm head is closely similar to that described for spermatozoa of the domestic ungulates (Blom \& Birch-Andersen, 1961; Nicander \& Bane, 1962; Kojima, 1962), but it should not be assumed that there are no differences in structure between the spermatozoa of these species. It appears, for instance, that the midpiece of the bull spermatozoon contains 70 to 100 mitochondrial coils (E. Blom, personal communication) and that of the boar about sixty-five coils (Nicander \& Bane, 1962: Fig. 7), whereas not more than about forty-seven coils are present in the mitochondrial helix of the 
rabbit spermatozoon (unpublished observations). It has been suggested that the apical fold and vacuole, seen in bull spermatozoa by Blom \& BirchAndersen (1961), may represent a normal structural arrangement of the acrosome cap in this species. While a similar structural form has also been observed in the acrosome of a few rabbit spermatozoa in samples from several different males (Pl. 2, Fig. 6), it is clear, from observations of central sagittal sections of many spermatozoa from these and other males, that the anterior acrosome region in the great majority of rabbit spermatozoa appears as a smooth swelling (Pl. I, Fig. 4). The question as to whether or not spermatozoa which possess the apical fold are able to penetrate the egg, obviously cannot be answered at present. It seems possible that the presence of an apical fold in rabbit spermatozoa represents an abnormality of development, akin to the Type I abnormality of boar spermatozoa described by Bane (1961). The enlarged cystic deformity (Pl. 2, Fig. 7) seen in a few rabbit spermatozoa in the same samples, is clearly a malformation of the acrosome similar to that described by Blom \& Birch-Andersen (1962), in bull spermatozoa, and differs somewhat from the Type II abnormality of Bane (1961), which appears also as an abnormality of nucleus formation. It is interesting, though perhaps not surprising, that similar types of developmental abnormalities should occur in rabbit, bull and boar semen. The reports of this cystic condition of the acrosome in the bull and boar derive from the examination of semen samples from animals presented initially because of infertility, in which the incidence of sperm abnormality was approximately 90\% (Teunissen, 1946; Hancock, 1949; Bane, 1961 ; Blom \& Birch-Andersen, 1962). In the rabbit semen examined in the present study, however, the abnormal forms were found in semen samples in which the majority of spermatozoa showed normal structural features and apparently also normal fertility. In view of these findings in the rabbit, it seems that extensive examination with the electron microscope of semen from male ungulates showing slightly lowered or even normal fertility might reveal the occurrence of this type of abnormality at a low level, in a greater percentage of animals than at present suspected.

In the present state of knowledge concerning the structure and function of mammalian spermatozoa, it is not possible to pass more than brief comment upon the significance of the apical body, which clearly forms a ridge over the leading edge of the sperm nucleus. Evidence has been presented to show that the apical body persists in rabbit spermatozoa penetrating the zona pellucida, in which the material of the acrosome cap has mostly disappeared (Hadek, 1963). Considering its obvious stability, it seems possible that the apical body may prove to be homologous with the rod of the perforatorium described in the spermatozoa of various rodents (Clermont, Einberg, Leblond \& Wagner, 1955; Austin \& Bishop, 1958a). Hadek (1963) has commented that in rabbit spermatozoa seen within the zona pellucida, the apical body appears to be elongated, compared to the same structure seen in ejaculate spermatozoa, or in epididymal spermatozoa adhering to the surface of the ovum following incubation in vitro. It must be pointed out, however, that the proportions of the apical body in spermatozoa prior to fertilization may vary considerably, depending on the angle of section and on the particular region sectioned. 
There exists an obvious and consistent difference in the disposition of the plasma membrane over different regions of the rabbit sperm head, and it seems also that the membrane overlying the acrosome cap shows greater adhesive properties than that over the post-nuclear cap or over the tail. The greater adhesiveness of the plasma membrane over the acrosome may be a reflection of different surface properties in this region; on the other hand, the looseness of the membrane over the acrosome may provide a means whereby the vigorous movements of the spermatozoa are damped or cushioned, so that once contact has been established the movements of the spermatozoa do not immediately overcome the attracting forces binding the cell surfaces. Considering the presence of small particles often seen to be adherent to the plasma membrane over the acrosome cap region only, it seems likely that both these factors may operate to ensure that the spermatozoa stick more readily by the acrosome region than by the remainder of the sperm surface.

\section{ACKNOWLEDGMENTS}

I thank Dr A. M. Lawn and Mrs Mary Shuttleworth for much help and advice on techniques, and Miss Gillian Tomlinson for her technical assistance. I would also like to thank Professor E. C. Amoroso, F.R.s., for his criticism of the manuscript.

This work was supported in part by United States Public Health Service grant RG6489 from the National Institute of Health, Bethesda, Maryland.

\section{REFERENCES}

Adams, C. E. \& Chang, M. G. (1962) Capacitation of rabbit spermatozoa in the Fallopian tube and uterus. F. exp. Zool. 151, 159.

Austin, C. R. (1951) Observations on the penetration of sperm into the mammalian egg. Aust. F. sci. Res. B, 4, 581.

Austin, C. R. (1961) Fertilization of mammalian eggs in vitro. Int. Rev. Cytol. 12, 337.

Austin, G. R. \& Bishop, M. W. H. (1958a) Some features of the acrosome and perforatorium in mammalian spermatozoa. Proc. roy. Soc. B, 149, 234.

Austin, C. R. \& Bishop, M. W. H. (1958b) Role of the rodent acrosome and perforatorium in fertilization. Proc. roy. Soc. B, 149, 241.

Bane, A. (1961) Acrosomal abnormality associated with sterility in boar. Proc. IVth int. Congr. Anim. Reprod., The Hague, p. 810.

BeDford, J. M. (1963) Morphological reaction of spermatozoa in the female reproductive tract of the rabbit. F. Reprod. Fertil. 6, 245.

BLom, E. \& BirCh-ANDERsen, A. (1961) An apical body in the galea capitis of the normal bull sperm. Nature, Lond. 190, 1127.

Blom, E. \& Birch-Andersen, A. (1962) Ultrastructure of the sterilizing "knobbed-sperm" defect in the bull. Nature, Lond. 194, 989.

Caulfield, J. B. (1957) Effects of varying the vehicle for $\mathrm{OsO}_{4}$ in tissue fixation. F. biophys. biochem. Cytol. 3, 827.

Chang, M. C. (1951) Fertilizing capacity of spermatozoa deposited into the Fallopian tubes. Nature, Lond. 168, 697.

Clermont, Y., Eingerg, E., Leblond, G. P. \& Wagner, S. (1955) The perforatorium-an extension of the nuclear membrane of the rat spermatozoon. Anat. Rec. 121, 1.

Fawcett, D. W. (1958) The structure of the mammalian spermatozoon. Int. Rev. Cytol. 7, 195.

GLAUERT, A. M. \& Glauert, R. H. (1958) Araldite as an embedding medium for electron microscopy. J. biophys. biochem. Cytol. 4, 191.

HADEK, R. (1963) Submicroscopic changes in the penetrating spermatozoon of the rabbit. $\mathcal{f}$. ultrastruct. Res. 8, 161. 
Hamner, C. E. \& Williams, W. L. (1963) Effect of the female reproductive tract on sperm metabolism in the rabbit and fowl. F. Reprod. Fertil. 5, 143.

HANCOCK, J. L. (1949) Evidence of an inherited seminal character associated with infertility of Friesian bulls. Vet. Rec. 61, 308.

Kojima, Y. (1962) Electron Microscopic Study of the Bull Spermatozoon. Doctorate thesis, Hokkaido University.

Millonig, G. (1961) A modified procedure for lead staining of thin sections. F. biophys. biochem. Cytol. 11, 736 .

Moricard, R. (1960) Observations de microscopie électronique sur des modifications acrosomique lors de la pénétration spermatique dans l'oeuf des mammifères. C.R. Soc. Biol., Paris, 154, 2187.

Nicander, L. \& Bane, A. (1962) Fine structure of boar spermatozoa. Z. Zellforsch. 57, 390.

OHNUKI, Y. (1959) Some morphological observations of mouse spermatozoa in relation to insemination. Zool. Mag., Tokyo, 68, 275. Biol. Abstr. (1960), 35, 46462.

Palade, G. E. (1952) A study of fixation for electron microscopy. J. exp. Med. 95, 285.

Robertson, J. D. (1960) The molecular structure and contact relationships of cell membranes. Progr. Biophys. 10, 343.

Teunissen, G. H. B. (1946) Een afkijking van het acrosoom (kopkap) bij de spermatozoiden van een stier. Tijdschr. Diergeneesk. 71, 292.

\section{EXPLANATION OF PLATES}

\section{PLATE 1}

Fig. 1. Non-central sagittal section of rabbit sperm head, incubated for $12 \mathrm{hr}$ in serumRinger before fixation. Note the typically loose plasma membrane, closely adherent over the apex of the acrosome cap and over the post-nuclear cap. The thickness of the acrosome cap decreases in the region of the 'equatorial segment' seen with the light microscope. Uranyl acetate and potassium permanganate. $\times 35,000$.

FIG. 2. Transverse section of rabbit sperm head, incubated in serum-Ringer for $12 \mathrm{hr}$ before fixation. Note the loose plasma membrane adherent at the edges of the sperm head, where the acrosome cap thickens considerably. Note also the asymmetry of nuclear shape. Uranyl acetate and potassium permanganate. $\times 36,000$.

Fig. 3. Sagittal sections of rabbit sperm head at the junction of the acrosome and post-nuclear caps. (a) The outer nuclear membrane appears as the inner limiting membrane of the post-nuclear cap. Note the presence of some material between the inner acrosome and outer nuclear membranes. Uterine spermatozoon: Lead hydroxide. $\times 100,500$. (b) Note the bilaminar appearance of the acrosome membrane, which contrasts with the single membrane seen in lead-stained section (a). Ejaculate spermatozoa incubated in serum-Ringer for $12 \mathrm{hr}$. Uranyl acetate and potassium permanganate. $\times 107,000$.

FIG. 4. Sagittal section of rabbit sperm head (uterine), showing the typical swollen apex with clearly defined apical body. Lead hydroxide. $\times 86,000$.

\section{PLATE 2}

FIG. 5. Sagittal section of rabbit sperm head (ejaculate). The acrosome cap is disintegrating but typically the inner acrosome membrane and apical body remain intact. Lead hydroxide. $\times 67,500$.

FIG. 6. Sagittal section of rabbit sperm head (uterine). The acrosome cap possesses a clearly defined anterior flap, which contrasts with the smooth swelling seen in Pl. 1, Fig. 4. Note the distortion of the plasma membrane in the region adhering to a uterine polymorphonuclear leucocyte. Lead hydroxide. $\times 44,200$.

FIG. 7. Sagittal section of anterior region of rabbit sperm head (ejaculate). This shows a terminal cystic structure bounded by an intact plasma membrane. Uranyl acetate and potassium permanganate. $\times 52,500$.

Fig. 8. Sagittal section of rabbit sperm head (uterine). This shows the situation in a number of uterine spermatozoa in which the plasma membrane becomes separated from the edge of the acrosome cap. Note the presence of particles on the outer surface of the plasma membrane, which is apparently adherent over a small area to the surface of a leucocyte. Lead hydroxide. $\times 51,500$. 
PLATE 1

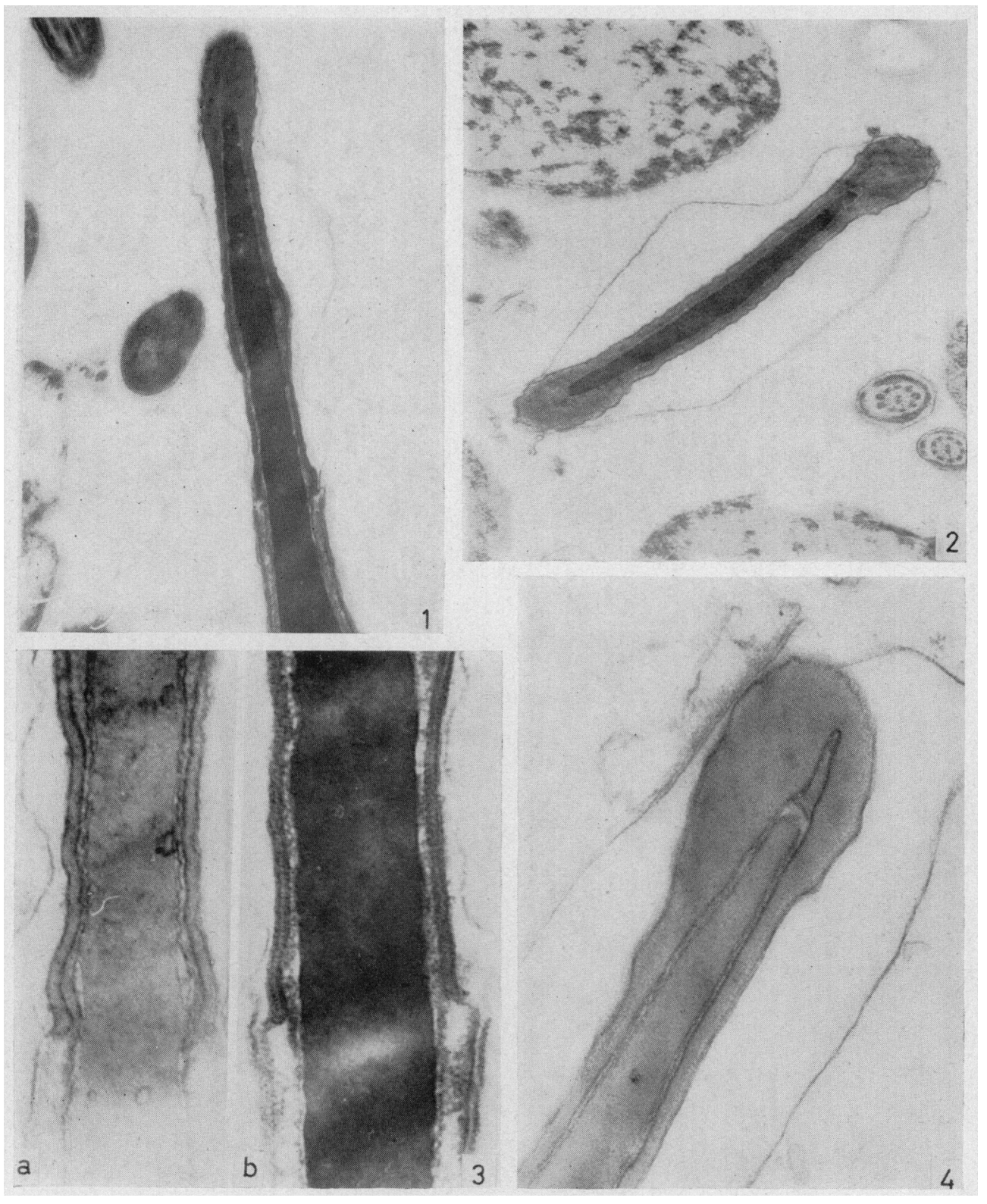

(Facing p. 228) 
PLATE 2

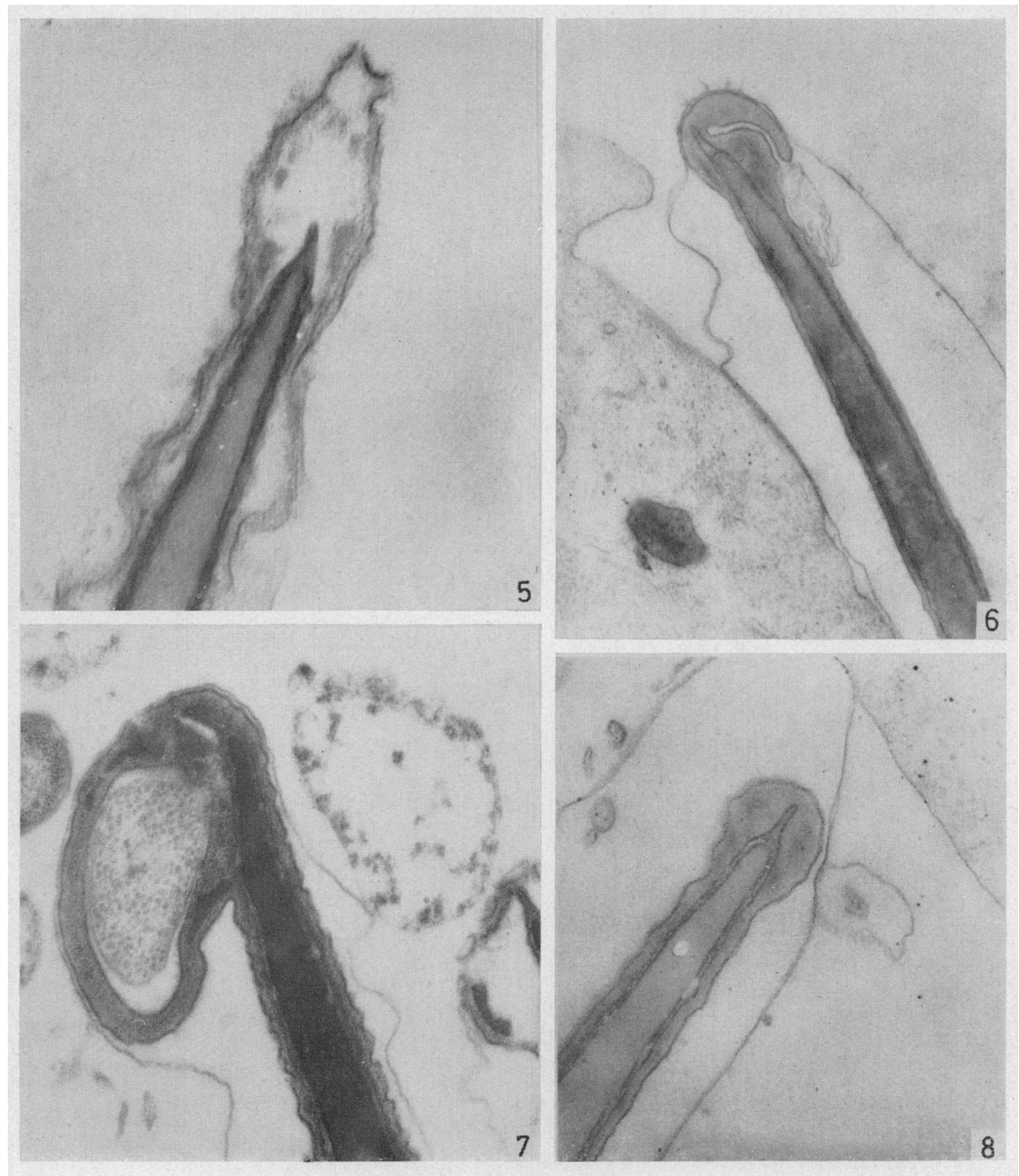

